\title{
Boundary Friction on Molecular Lubricants: Rolling Mode?
}

\author{
V.M. Loktev ${ }^{1}$ and Yu.G. Pogorelov ${ }^{2}$ \\ ${ }^{1}$ Bogolyubov Institute for Theoretical Physics, National Academy of Sciences of Ukraine, \\ Metrologichna Str. 14-b, Kiev, 03143 Ukraine, ${ }^{2}$ CFP/Departamento de Física, \\ Universidade do Porto, 4169-007 Porto, Portugal
}

(Dated: November 2, 2018)

\begin{abstract}
A theoretical model is proposed for low temperature friction between two smooth rigid solid surfaces separated by lubricant molecules, admitting their deformations and rotations. Appearance of different modes of energy dissipation (by "rocking" or "rolling" of lubricants) at slow relative displacement of the surfaces is shown to be accompanied by the stick-and-slip features and reveals a non-monotonic (mean) friction force vs external load

PACS numbers: $46.55 .+\mathrm{d}, 81.40 . \mathrm{Pq}$
\end{abstract}

\section{INTRODUCTION}

In the modern tribology a still increasing interest is put to the studies of wearless friction on atomically smooth surfaces [1, 2] as a possibility to provide an information about the basic processes of energy losses on microscopic level, important for the purposes of optimization in many technological applications. This is also connected with the search for best coating and lubricant materials. The principal physical picture, usually considered in relation with boundary friction on few molecular layers of lubricant liquid, is the sequence of "freezing-melting" processes on the lubricant, giving rise to discontinious (stickand-slip) displacement of sliding surfaces [3]. Recently a new theoretical approach was proposed for microscopic sliding processes at extremely low velocities of motion and upon a monolayer of lubricant atoms [4], as can be the case for friction force microscopy (FFM). Based on the adiabatic formation of metastable states (similar to dislocations in usual deformed crystals or defects in the Frenkel-Kontorova model [], , $\underline{6}]$ ) and their following relaxation, this treatment shows how the (average) microscopic friction coefficient depends upon the material parameters of the contacting solids and lubricants and also how stick-and-slip jumps with atomic periodicity can develop in the microscopic friction force. It should be noted that a similar microscopic mechanism of dislocation-assisted sliding was recently proposed for contacting asperities in dry friction [7].

Ultimately, with the discovery of almost spheroidal molecule of fullerene $\mathrm{C}_{60}$ [8] (and/or cylindrical carbon nanotubes [9]) a hope had arosen to use such closed molecular structures, as "free rotating" lubricants, for considerable reduction of the friction coefficient. For instance, a reduction of sliding friction coefficient was already discussed, due to involvement of spinning motion of surfaces in contact [10]. However, the FFM experiments with use of $\mathrm{C}_{60}$ monolayers deposited over atomically smooth solid surfaces brought some contradictory results 11], 12], 13]. To get their better understanding, a further theoretical insight is desirable on the elementary processes of boundary friction.

The present communication is aimed on extention of the above mentioned adiabatic approach to the processes of boundary friction which include the internal degrees of freedom of the lubricant molecules. Within a simplest model, we make an attempt to show that, due to the discrete atomic structure of such a molecule, there are possible qualitatively different modes of slow motion, either dissipative or non-dissipative, depending on the applied external load on the contact. For dissipative modes, there are energy losses resulting from stick-and-slip discontinuities, but these losses turn out much lower then for similar processes at sliding solid surfaces upon atomic lubricants. Besides, the mean value of friction force is found to be a non-monotonic function of the external load.

\section{THE MODEL}

Let us consider a two-dimensional model for the boundary friction system which includes two semi-infinite atomic arrays, the "solids", with identical triangular lattice structure and a spatial separation $d$ between their srufaces, and clusters of four atoms, the "molecular lubricants", confined between the solids (Fig. 1). The distances between nearest neighbour atoms in both solids and in clusters are supposed invariable 14] (that means, corresponding to "absolutely rigid" bonds), and the bond length for the molecule equals to the lattice parameter $a$ for the solid surfaces.

The model includes certain "soft links", which determine the energy variation $v s$ relative displacement of the solids. In the first turn, this is weak Lennard-Jones interaction between an atom of solid and a lubricant molecule at distance $r$ :

$$
V_{L J}(r)=\varepsilon_{0}\left[\left(\frac{r_{0}}{r}\right)^{6}-2\left(\frac{r_{0}}{r}\right)^{12}\right],
$$

characterized by the adhesion energy $\varepsilon_{0}$ and equilibrium distance $r_{0}$. Besides, a lubricant molecule can experience 


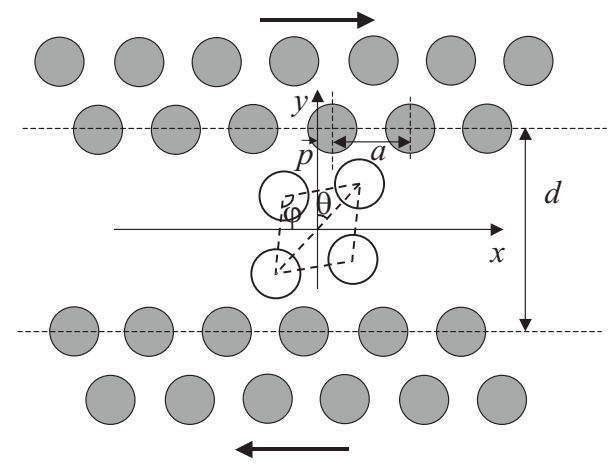

FIG. 1: Schematic of the friction system: two solid arrays (grey circles) with identical lattice structures are separated by a four-atomic lubricant molecule (white circles). The molecule center rests at the origin, and its rhombic configuration is determined by the angles $\theta$ and $\phi$, while the symmetric displacements of solids are described by the parameter $p$ (see the text).

rhombic deformation which changes its energy as $k \sin ^{2} \phi$, where $k$ and $\phi$ are the elastic constant and rhombic angle, respectively. We neglect the interaction between different lubricants, that is omit their possible collective modes. Thus the molecular lubricants are supposed to contribute independently into the total friction force. Then we choose one particular molecule and set its center of mass as the origin of reference frame, so its configuration is determined by the "internal" rhombic angle, $\phi$, and the "external" orientation angle, $\theta$ (say, with respect to the normal to interface). In such a frame, the two solids are supposed to be displaced symmetrically with respect to the lubricant molecule and their configuration is fully defined by the displacement parameter $p$ chosen, e.g., as the smallest positive longitudinal coordinate of the atoms of upper solid.

All this permits to write the full energy (per one lubricant) in the simple form:

$$
E=\sum_{n, m} V_{L-J}\left(\left|\mathbf{r}_{n}-\mathbf{R}_{m}\right|\right)+k \sin ^{2} \phi
$$

where $\mathbf{r}_{n}$ and $\mathbf{R}_{m}$ are respectively positions of atoms of solids and atoms in the lubricant molecule. Obviously, this extremely simplified model does not pretend to give a quantitative description and explanation of friction experiments in real systems with complex and typically incommensurate solid and molecular structures. It serves mainly to illustrate some new qualitative possibilities for weakly dissipative processes, associated with the internal (rolling or rotational) degrees of freedom of molecular (nanotubes including) lubricants, in contrast to the dissipation by only translational motions of the "point-like" (atomic) lubricants.

\section{THE ADIABATIC DYNAMICS}

The adiabatic treatment of the system, corresponding to the expression (2), follows the lines suggested in Ref. [4]. The equilibrium distance for Lennard-Jonnes interaction (11) is taken equal to the interatomic distance in the solids: $r_{0}=a$. At given separation $d$ between the surfaces, we calculate numerically the total energy profile $E(p, \theta, \phi)$ as a function of the displacement parameter $p$, and also of the angles $\theta$ and $\phi$. Next this function is optimized with respect to the deformation angle $\phi$ to result in the profiles $E(p, \theta)$, such as displayed in Figs. 25 (for different values of $p$, through the whole displacement period from 0 to $a$, and at different separations $d$ ).

The primary optimization in $\phi$ refers to the stronger elastic deformation constant (we took $k=0.5 \varepsilon_{0}$, while the amplitude of relevant energy oscillations in $\theta$ is $\left.\sim 0.1 \varepsilon_{0}\right)$, and hence to a faster relaxation in $\phi$ than in $\theta$. 15. Then the system behavior at very slow uniform variation of the parameter $p$ with time (that is, the slow dynamics) is obtained from the analysis of the profile $E(p, \theta)$. Below we analyze how this profile changes with growing external load, which is here simulated by a gradual decrease of the separation distance $d$.

i) At greatest separations (evidently corresponding to the lowest loads), it is seen from Fig. 22 that the energy profile has a single $\pi / 2$ periodicity in $\theta$. This means that, for any given $p$, there is a single equilibrium state for the molecule, characterized by its orientation $\theta(p)$ (within to a $C_{4}$ rotation) and energy $E(\theta(p))$, such that $(\partial E / \partial \theta)_{\theta(p)}=0,\left(\partial^{2} E / \partial \theta^{2}\right)_{\theta(p)}>0$. In this case, the phase trajectory $E(\theta)$ (shown by the sequence of bold dots linked by arrows, for growing displacement) is closed and continious. Thus, the system energy changes in a fully reversible way and, though some forces are exerted in the process, their mean value over the cycle and so the mean friction force are exactly zero. This reversible variation of the angle $\theta$ around its median position (such that the long diagonal of the rhomb points vertically, inset to Fig. 21), corresponds to a "rocking mode" of the molecular motion.

ii) When the two solid surfaces are getting closer (at increasing load), the first qualitative change that appears in the system behavior is the doubling of its energy minima. Now there are two splitted minima at the initial configuration $p=0$ (Fig. 3), which correspond to a two-fold degenerate equilibrium state of the deformed lubricant molecule: the long diagonal of the rhomb can deviate by a finite angle $<\pi / 2$ to both sides from the vertical (inset to Fig. [3). Since the molecule is considered a classic object, it initially occupies only one of the minima (the left one is chosen in Fig. 31).

However the degeneracy gets lifted for $p>0$, so that one of the splitted minima turns stable $(s)$ and another a metastable $(m)$ equilibrium state. As it is seen from 


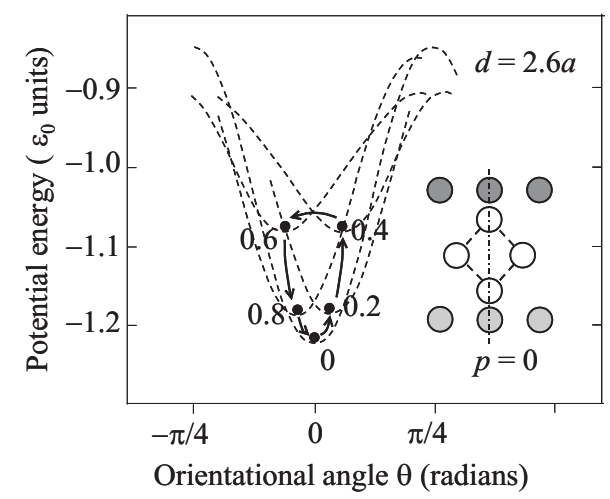

FIG. 2: Series of profiles of potential energy vs orientation (at optimal deformation, see the text) and the related equilibrium states (bold circles) of a lubricant molecule at growing displacement of the solids (the numbers indicate $p / a$ values) and at highest separation between them. The phase trajectory (arrows) shows that the molecule performs continuous "rocking" motions and after the full cycle (at $p / a=1$ ) returns to its initial state with no energy dissipation. Inset: the rhombic configuration at $p=0$.

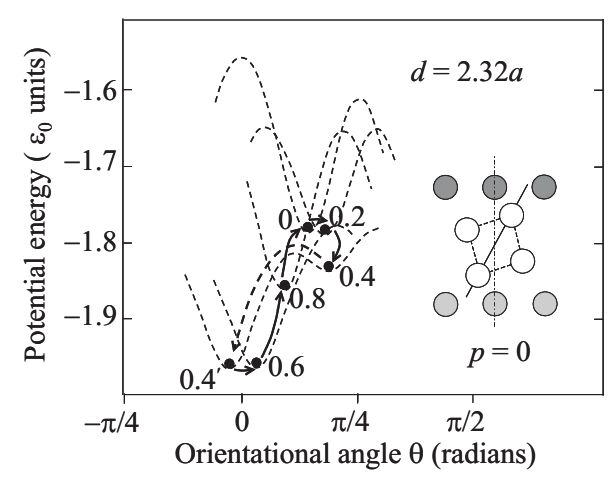

FIG. 3: The same as in Fig. 2]but at closer separation between the solids (higher external load). The lubricant molecule after "rocking" returns to its initial state, but in this course it experiences a thermally activated jump (the dotted arrow over the potential barrier for $p / a=0.4$ ) between the metastable (0.4) and stable $\left(0.4^{\prime}\right)$ minima, and the corresponding energy difference is irreversibly lost. Inset: the configuration at $p=0$ corresponds to one of the splitted minima

the consecutive curves in Fig. 3] the energy barrier $h$ between the $m$-state and the nearest $s$-state (here that to the left from $m$ ) decreases by many times with growing $p$, as a certain function $h(p)$. Since the adiabatic lifetime for the $m$-state is: $\tau_{m}=\tau_{a} \exp (\beta h)$ (where $\tau_{a} \sim 10^{-12} \mathrm{~s}$ is the atomic oscillation time and $\beta$ is the inverse temperature), it decreases in this course by many orders of magnitude. Eventually, this lifetime gets comparable to the characteristic time $\tau_{0}$ of slow displacement by an atomic period, at a very sharply defined instant when $p=p_{0}$ so that $h\left(p_{0}\right)=h_{0}=\beta^{-1} \ln \left(\tau_{0} / \tau_{a}\right)$. Hence it is almost exactly at this instant that a thermally activated jump from $m$ - to $s$-state is realized. After the jump, the en-

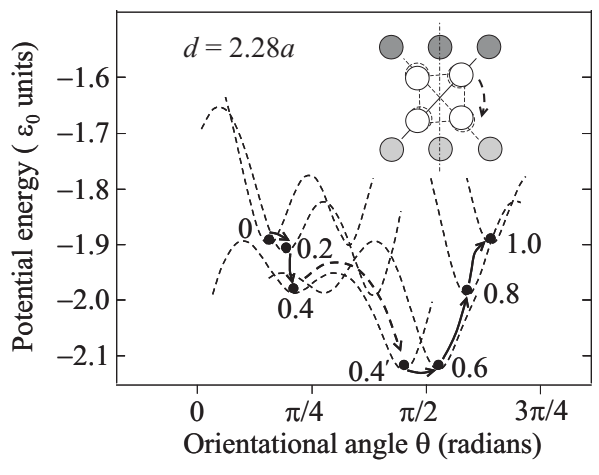

FIG. 4: The same as in Figs. 2 and 3 but at still higher load. The thermally activated jump changes its direction and the lubricant molecule rotates by the angle $\pi / 2$ after every period in $p$ ("rolling mode"). The energy loss and hence the friction force is somewhat larger than that in Fig. 3

ergy difference between $m$ - and $s$-states (to the moment of transition) is irreversibly lost, through the creation of quasiparticles (phonons, for insulating solids) which are finally thermalized in the bulk. For typical displacement rates in FFM $\sim 10^{2} \rho \mathrm{A} / \mathrm{s}$, one has $\tau_{0} \sim 10^{-2} \mathrm{~s}$, so that the barrier to the transition moment is still as high as

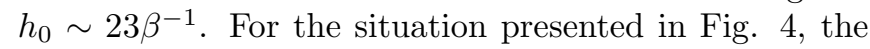
transition corresponds to $p_{0}=0.4 a$ (the potential profile shown by the solid line). It is also seen that, with further growing $p$, up to $a$, the system returns to its initial state; thus the phase trajectory is still closed ("rocking mode" again) though discontinious. This discontiniuity produces stick-and-slip features in the microscopic behavior of the force vs displacement and it is the only source of the irreversible losses in our model system, in a full similarity to the model of sliding solids upon atomic lubricants.

There are however some distinctions between the two systems. Firstly, the stick-and-slip profile for molecular lubricants is more complicated than the simple triangular sawtooth for atomic lubricants. But especially important is the fact that the irreversible forces for molecular lubricants are smaller than elastic, reversible forces (the jump heights in Figs. 35 are noticeably lower then the amplitudes of smooth oscillations), while the irreversible forces for atomic lubricants are orders of magnitude higher than the reversible ones. Since the reversible forces in both cases are characterized by the same energy scale $\varepsilon_{0}$, this indicates a possibility to essentualy reduce the dissipation by molecular (rotating) lubricants $v s$ that by atomic lubricants. Note, at least, that if the molecule occurs initially (at $p=0$ ) in the right minimum, it simply spends a period, $0<p<a$, in the $s$-state, but then passes to the $m$-state during the next period and eventually comes to the same closed discontinuous rocking.

iii) Now let us bring the surfaces yet a little bit closer, as by a very small change of the distance $d$ between the 


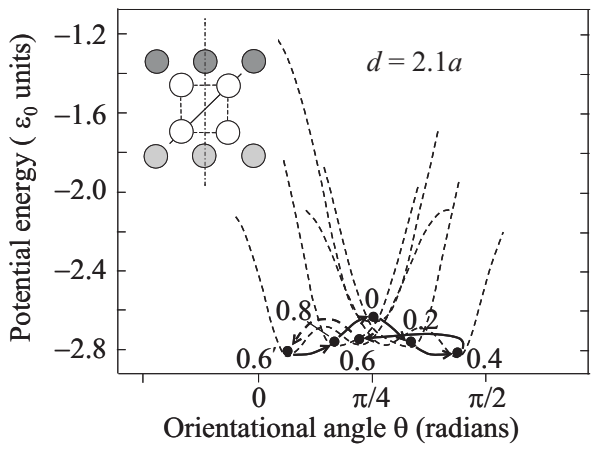

FIG. 5: The same as in Figs. 2 4 at futher growing load. The molecule returns from "rolling" to "rocking" regime with an intermediate jump, but at much smaller energy loss than in Figs. 3 and 4

solids (Figs. 3 and 4). Then the system evolution acquires yet a new quality: now at the transition moment the nearest $s$-state is to the right from the given $m$-state. This results in that after the transition, at $p>p_{0}$, the molecule does not return to its initial state, but will be eventually rotated by the angle $\pi / 2$ (the dashed circles and arrow in the inset to Fig. 4), and this rotation will be repeated each next period. Thus, in this case we have both unclosed (corresponding to the "rolling mode") and discontinious regime of lubricant motion.

$i v)$ With still increasing loads, the situation of a single minimum for $E(0, \theta)$ will be restored again (though displaced by the angle $\pi / 4)$, corresponding to a "square" molecule (inset to Fig. 5). But now, unlike the case of low loads, this minimum gets splitted with increasing displacement $p$ (here at $p \approx 0.3$ ). The molecule returns from "rolling" to "rocking" regime ("rock-n-roll dance"), but with considerably reduced energy dissipation: the energy loss at a jump for $d=2.1 a$ (Fig. (5) is about 2.5 times smaller than for $d=2.28 a$ (Fig. 4), i.e. it decreases with load increasing. We do not present here detailed results of numerical simulations for even higher loads, resuming only that they reveal a number of subsequent dissipative regimes, either with growing and falling friction forces.

\section{CONCLUSIONS}

The above simple analysis demonstrates that boundary friction with participation of molecular (spheroidal or cylindrical) lubricants can possess quite unusual properties, such as existence of various regimes of molecular motion, either non-dissipative and dissipative, with abrupt transitions from one regime to another at continious variation of external load. In the sequence of regimes ("rocking" and "rolling"), the obtained friction force vs load displays non-monotonic and hence non-linear behavior. This model (of course, with due improvements to be inserted) may provide a mechanism for explaining the data of real experiments with the fullerenes $C_{60}$ as lubricants and give indications for an optimal regime of their practical applications.

\section{Acknowledgments}

V.M.L. acknowledges partial support of this investigation by CRDF grant SCOPES 7UKPJ062150.00/1.

[1] B. Bhushan, J.N. Israelashvili, and U. Landman. Nature 374, 607 (1995).

[2] G.M. McClelland, and J.N. Glosli. In: Fundamentals of Friction: Macroscopic and Microscopic Processes /Eds. I.L. Singer and H.M. Pollock (Kluwer, Dordrecht, 1992). P. 405.

[3] J.N. Israelachvili. Intermolecular and Surface Forces, Academic Press, London (1991).

[4] Yu.G. Pogorelov. Preprint cond-mat/0309117 (2003).

[5] Y.I. Frenkel and T. Kontorova, Zh. Eksp. Teor. Fiz., 8, 1340 (1938).

[6] J.B. Sokoloff, Phys. Rev. B42, 760 (1990).

[7] B. Bhushan and M. Nosonovsky. Acta Mater. 51, 4331 (2003).

[8] H. Kroto, J.R. Heath, S.C. O'Brian, R.F. Curl, and R.E. Smalley. Nature 318, 162 (1985).

[9] S. Iijima. Nature 354, 56 (1991).

[10] Z. Farkas, G. Bartels, T. Unger, and D.E. Wolf. Phys. Rev. Lett. 90, 248302 (2003).

[11] B. Bhushan, B.K. Gupta, G.W. van Geef, C. Capp, and J.V. Coe. Appl. Phys. Lett. 62, 3253 (1993).

[12] R. Luthi, E. Meyer, H. Haefke, L. Howald, W. Gutmannsbauer, M. Guggisberg, M. Bammerlin, and H.-J. Guntherodt. Surface Sci. 338, 247 (1995).

[13] U.D. Schwarz, W. Allers, G. Gensterblum, and R. Wiesendanger. Phys. Rev. B 52, 14976 (1995).

[14] Here mean distances are meant, as the only relevant for adiabatic equilibrium states, while small thermal fluctuations become important for activation of relatively fast transitions between these equilibrium states.

[15] Similar hierarchy of relaxation rates between two degrees of freedom was also reported for point-like lubricants, corresponding to their normal (transversal) and longitudinal displacements [4]. 